\title{
Gender Influences on Shoulder Arthroplasty
}

\author{
Nicole J. Hung ${ }^{1}$ - Stephanie E. Wong ${ }^{2}$ (1) \\ Accepted: 22 November 2021 / Published online: 4 January 2022 \\ (C) The Author(s) 2021
}

\begin{abstract}
Purpose of Review As the incidence of shoulder arthroplasty continues to increase, there is growing interest in patient-based factors that may predict outcomes. Based on existing literature demonstrating gender-based disparities following total hip and knee arthroplasty, gender may also influence shoulder arthroplasty. The purpose of this review is to discuss the recent literature on the influence of gender on shoulder arthroplasty, focusing on differences in preoperative parameters, perioperative complications, and postoperative outcomes.

Recent Findings While both female and male patients generally benefit from shoulder arthroplasty, several differences may exist in preoperative factors, acute perioperative complications, and postoperative outcomes. Preoperatively, female patients undergo shoulder arthroplasty at an older age compared to their male counterparts. They may also have greater levels of preoperative disability and different preoperative expectations. Perioperatively, female patients may be at increased risk of extended length of stay, postoperative thromboembolic events, and blood transfusion. Postoperatively, female patients may achieve lower postoperative functional scores and decreased range of motion compared to male patients. Differences in postoperative functional scores may be influenced by gender-based differences in activities of daily living. Finally, female patients may be at greater risk for periprosthetic fracture and aseptic loosening while male patients appear to be at greater risk for periprosthetic infection and revision surgery.

Summary Current literature on the influence of gender on shoulder arthroplasty is limited and conflicting. Further research is necessary to delineate how gender affects patients at the pre- and postoperative levels to better inform decision-making and outcomes.
\end{abstract}

Keywords Total shoulder arthroplasty $\cdot$ Reverse shoulder arthroplasty $\cdot$ Gender $\cdot$ Outcomes $\cdot$ Disparities $\cdot$ Complications

\section{Introduction}

Shoulder arthroplasty is an effective treatment for shoulder pathology including glenohumeral osteoarthritis $(\mathrm{OA})$, rotator cuff arthropathy, and proximal humerus fractures. Since Péan performed the first documented shoulder arthroplasty in 1894, there have been significant advances in implant development and surgical technique [1]. In 1955, Neer developed the first modern total shoulder arthroplasty (TSA) to treat proximal

This article is part of the Topical Collection on Gender and Racial Disparities

Stephanie E. Wong

swong@ucsf.edu

1 School of Medicine, University of California, San Francisco, San Francisco, CA, USA

2 Department of Orthopaedic Surgery, University of California, San Francisco, 1500 Owens Street, San Francisco, CA 94158, USA humerus fractures, reporting pain relief in patients who received a proximal humerus prosthesis [2]. There has been a rapid rise in shoulder arthroplasty utilization since 2004, which is partially attributed to the introduction and approval of a reverse total shoulder arthroplasty (RTSA) device by the Food and Drug and Administration (FDA) in November 2003 [3]. RTSA was originally introduced as an effective surgical treatment of rotator cuff arthropathy, but its indications have since expanded to include treatment of proximal humerus fractures, revision shoulder arthroplasty, and tumors [4]. Overall, shoulder arthroplasty is a successful procedure that leads to pain relief and improved function [5•,6].

Although the exact incidence of glenohumeral OA is unknown, cadaveric and radiographic studies have demonstrated evidence of glenohumeral OA in up to $32.8 \%$ of adults over the age of $60[7,8]$. As the American population continues to age, the incidence of shoulder arthroplasty will continue to increase. Demand for shoulder arthroplasty is estimated to increase by $355-755 \%$ by $2030[3,9,10]$. In fact, demand 
for shoulder arthroplasty may overcome that for total hip arthroplasty (THA) and total knee arthroplasty (TKA) [10]. Given the projected increase in shoulder arthroplasty, it is important to consider patient factors that may impact postoperative outcomes.

Previous studies have described disparities in outcomes for total hip and knee arthroplasty patients based on gender [11-14]. These differences in outcomes may be impacted by female patients having a worse preoperative functional status before undergoing THA and TKA [15]. Although limited, current literature suggests that gender also influences outcomes for patients undergoing shoulder arthroplasty. The purpose of this review is to discuss the recent literature on the influence of gender on shoulder arthroplasty, focusing on differences in preoperative parameters, perioperative complications, and postoperative outcomes.

\section{Differences in Preoperative Parameters}

Several studies have described differences in preoperative factors between male and female patients undergoing shoulder arthroplasty. These factors include patient age at presentation, preoperative disability level, and preoperative patient expectations. Female patients typically undergo shoulder arthroplasty at an older age compared to male patients [16, $17 \cdot 0$. In a recent study, Okoroha et al. reported an average age at time of TSA and RTSA in female versus male patients of $71.2 \pm 8.5$ years versus $67.4 \pm 8.9$ years $(p<.01)[17 \bullet \bullet]$. Jawa et al. showed a similar trend in patients undergoing TSA as female patients had a mean age of at time of surgery of 66.4 years versus 60.8 years for male patients $(p=0.01)$ [16]. In a study by Wong et al., the mean age of the female cohort undergoing RTSA trended towards being greater than that of the male cohort (70.3 years versus 66.9 years, $p=0.078$ ) $[18 \bullet \bullet]$.

There is mixed evidence on whether female patients undergoing shoulder arthroplasty have greater levels of preoperative disability and lower baseline functional status. In a recent prospective study, Wong et al. found similar baseline range of motion in all planes in female and male patients undergoing RTSA [18••]. Additionally, preoperative 12-Item Short Form Health Survey (SF-12), American Shoulder and Elbow Surgeons Score (ASES), and Visual Analog Score (VAS) pain scores were similar between female and male patients [18••]. Jawa et al. similarly found no difference in preoperative ASES, SF-12, and VAS scores between female and male patients undergoing TSA.

In contrast, Okoroha et al. recently reported significantly lower preoperative clinical outcome scores (ASES, Constant, Simple Shoulder Test (SST), $p<0.01$ ) in female patients compared to male patients undergoing TSA and RTSA. This study also described decreased range of motion in female patients preoperatively, specifically decreased active abduction $(p<0.01)$, forward flexion $(p<0.01)$, and external rotation $(p=0.02)[17 \bullet \bullet]$. In a retrospective review of an international multicenter database of 660 patients undergoing RTSA, Friedman et al. similarly found lower preoperative clinical outcome scores for female patients (SST, ASES, Shoulder Pain and Disability Index (SPADI), and Constant, $p<$ $0.001)$ as well as more limited motion in abduction $(p=$ $0.001)$ and passive external rotation $(p<0.001)$ [19••].

Female and male patients who undergo shoulder arthroplasty appear to have different preoperative expectations. In a prospective study of 63 patients undergoing TSA by Jawa et al., male patients identified participation in sports, sleeping through the night, and maintaining employment as the top three preoperative expectations. On the other hand, female patients selected ability to independently perform household chores and daily routine as the top consideration, which differed significantly from men $(p<0.01)$. Despite this difference, female patients also valued sleeping through the night and exercising/participating in sports [16]. According to Henn et al., additional preoperative expectations that were more important to female patients than male patients undergoing TSA included stopping further shoulder dislocation $(p<$ $0.05)$, improving psychological well-being $(p<0.05)$, and improving driving ability or ability to put on a seatbelt $(p<$ 0.05) [20]. Additionally, Mancuso et al. previously described improvement in ability to provide self-care and complete pain relief as preoperative expectations of female patients undergoing shoulder surgery [21].

\section{Differences in Acute Perioperative Outcomes}

Existing literature suggests that female patients are at an increased risk of prolonged hospitalization after undergoing shoulder arthroplasty; however, recent studies challenge these findings [22-25]. Through large national database analysis, Menendez et al. found that female sex is associated with extended length of stay following $\mathrm{RTSA}(\mathrm{OR}=1.26, p=0.001)$ and TSA (OR $=2.14, p<0.001$ ) [25]. Dunn et al. reported similar findings with male patients requiring shorter length of stay after TSA (OR, 0.44 [95\% CI: 0.29, 0.66]; $p<0.0001$ ) [24]. Additionally, Matsen et al. analyzed 17,311 patients from the New York Statewide Planning and Research database and found that female sex is associated with longer hospitalization for all shoulder arthroplasty procedures including hemiarthroplasty, TSA, and RTSA [26].

In contrast to these studies, Okoroha et al. did not find an association between female sex and increased length of stay following shoulder arthroplasty [17••]. Wong et al. similarly found no difference in length of stay between female and male patients after RTSA (men, 2.32 days; women, 2.58 days; $p=$ 0.18 ) [18••]. Possible explanations for the differences in these 
findings include the confounding effect of study demographic (Matsen had 63\% women and Wong had 51.3\% women) and different types of arthroplasty included in the different analyses. Furthermore, $23.6 \%$ of shoulder arthroplasty in the Matsen study was performed for fracture, which was the diagnosis associated with the longest hospital stay [26].

Additional perioperative complications to consider include thromboembolic events, blood transfusions, short-term readmission, and opioid use. While female patients do not appear to be at increased risk of short-term readmission or increased inpatient opioid use after shoulder arthroplasty, there is an association between female sex and increased risk of thromboembolic events and blood transfusion after shoulder arthroplasty [18, 22, 26, 27]. Matsen et al. found no differences in risk of 90-day readmission between female and male patients after hemiarthroplasty, TSA, and RTSA [26]. Wong et al. found no difference in postoperative inpatient opioid use between female and male patients after RTSA [18••]. In a prospective study including 3480 patients undergoing TSA, Singh et al. demonstrated that female gender is associated with a significantly higher risk of 90-day thromboembolic event after TSA $(p<0.03)$ [22]. Finally, Gruson found female sex to be an independent risk factor for blood transfusion (OR 2.22; 95\% CI, 1.03-5.81; $p=0.04$ ) [26].

\section{Differences in Postoperative Outcomes}

Although female and male patients both appear to benefit from shoulder arthroplasty in pain relief and ROM, disparities in postoperative functional outcomes may exist [16-19, 28]. Okohora et al. showed no difference in postoperative satisfaction between female and male patients after shoulder arthroplasty with $90 \%$ of each cohort reporting to be "better" or "much better" postoperatively. Additionally, both cohorts showed significant postoperative improvement in all outcome measures. Yet, compared to male patients undergoing shoulder arthroplasty, female patients started and ended with lower outcome scores and range of motion [17••]. Furthermore, male sex has been associated with better outcome scores and ROM for all measurements except external rotation after RTSA $[19 \bullet \bullet]$.

In contrast, Wong et al. found comparable improvements in pain and ROM after RTSA for male and female patients; however, male patients had higher postoperative ASES function and SF-12 PCS scores at 1- and 2-year follow-up. For SF12 PCS scores, female patients only improved 69\% (1-year follow-up) and $42 \%$ (2-year follow-up) as much as the male patients. A similar trend in improvement was noted with ASES function scores at 1- (94\%) and 2-year (75\%) followup $[18 \bullet \bullet$.

Findings from several older studies also suggest worse postoperative functional outcome in female patients [28, 29].
Donigan et al. showed that female patients had lower SST scores postoperatively compared to male patients after TSA ( $p=0.0001)$; however, this finding may be confounded by the inclusion of hemiarthroplasties and patients with rheumatoid arthritis [16, 28]. Matsen et al. found that the strongest correlates with postoperative shoulder function were male gender $(p<0.0001)$, social function $(p<0.0001)$, mental health $(p<$ $0.0001)$, and preoperative shoulder function $(p<0.0001)$ [29]. Contrary to these findings, Jawa et al. found no differences between men and women in ASES/SF-12 at 3-year follow-up after TSA [16].

Differences between female and male patients after undergoing shoulder arthroplasty may also exist in rates of implantrelated failure, periprosthetic fracture, and periprosthetic infection. Okoroha et al. found that female patients were more likely to experience implant loosening $(p=0.03)$ and periprosthetic fracture $(p=0.01)$ while male patients had a higher incidence of periprosthetic infection $(p<0.01)$. However, the overall complication rate between the two cohorts was similar $(3.2 \%$ female vs. $3.6 \%$ male, $p=0.61)$ $[17 \bullet \bullet]$.

Male patients may be at higher risk of revision after shoulder arthroplasty. An older German registry study showed that male sex was associated with a higher revision burden compared to that of female patients following TSA [30]. Similarly, in an analysis of factors for revision TSA using the Mayo Clinic Total Joint Registry, Singh et al. found male sex to be significantly independently associated with increased risk of revision (HR $1.72(1.28,2.31), p<0.001)$ [31]. Contrary to these studies, Matsen et al. did not find male sex to be associated with an increased risk of revision following TSA and hemiarthroplasty; however, an analysis was not stratified with respect to sex for the different procedures [26].

\section{Conclusions}

Both female and male patients typically benefit from shoulder arthroplasty. Yet, differences in preoperative factors, acute perioperative complications, and postoperative outcomes may exist. Female patients typically undergo shoulder arthroplasty at an older age compared to male patients. They may also have worse preoperative disability and different preoperative expectations compared to male patients undergoing shoulder arthroplasty. Female patients may place greater importance on performing ADLs and household chores compared to male patients when considering preoperative expectations.

Age, preoperative disability, and preoperative expectations are likely interconnected. Age has been shown to influence preoperative expectations and worsen preoperative disability levels [20, 21]. Typically, younger patients place higher value on participation in sports, contributing to differences observed 
in preoperative expectations. Furthermore, the question of why female patients present at a later age may be related to differences in utilization of joint replacement between female and male patients. Although conflicting, existing literature suggests patient willingness to undergo surgery may influence utilization rates [32,33]. Qualitative studies have shown that female patients with hip and knee OA are often more accepting of greater functional decline over time compared to their male counterparts. Additionally, female patients may be less accepting of disrupting their roles and responsibilities as family caregivers to undergo surgery [32]. Another study cited increased concern of female patients about postoperative recovery as a contributor to decreased utilization of TKA [33]. On the other hand, in a survey of 48,218 patients evaluating willingness to undergo total knee arthroplasty (TKA) and total hip arthroplasty (THA) (response rate of 72\%), Hawker et al. found that female patients were three times less likely to undergo TKA/THA after controlling for willingness to undergo surgery [11]. These considerations may be similar in female patients who are contemplating shoulder arthroplasty, which could lead to later presentation and lower utilization rates.

In terms of acute perioperative complications, older studies suggest that female patients have increased length of stay following shoulder arthroplasty, but findings from more recent studies contradict this trend. These differences may be due to confounding factors such as demographics and inclusion criteria of the older studies. For example, in Matsen's study, hemiarthroplasty was included in procedure types, and proximal humerus fractures constituted a greater percentage of surgical indications; this diagnosis was independently associated with increased length of stay [26]. Additionally, female patients appear to be at an increased risk of blood transfusion and acute thromboembolic events whereas readmission and inpatient opioid use rates between female and male patients are comparable. This trend reflects previous findings' robust data regarding increased bleeding risk and transfusion rate in female patients undergoing elective procedures [34].

While both female and male patients benefit from shoulder arthroplasty similarly in pain score improvement, there are apparent differences in ROM improvement and postoperative functional scores. Existing literature has shown a trend towards lower postoperative functional scores and possibly less improvement in ROM postoperatively in females. However, a recent prospective study contradicted these findings, reporting similar postoperative ASES and SF-12 scores after TSA [16]. As Wong et al. noted, when considering the reason for apparently lower functional scores in females despite similar improvement in pain scores, it is important to consider how female patients may experience greater functional impairment based on differences in activities of daily living (ADLs). For example, brushing longer hair requires external rotation and donning a bra requires more internal rotation. Inability to perform these tasks may lead to lower functional scores [18••].
Based on this assessment, Lynch proposed that future research should include a formal analysis across genders of reliability, responsiveness, variability, and validity of commonly used patient-reported outcome measures [35•]. This poses the question of whether a new validated means of measurement is potentially needed given differences in ADLs between female and male patients. Finally, we should consider how improvements in these outcome scores translate clinically between male and female patients. Simovitch et al. found that female patients required a lower minimally clinically important difference (MCID) in all outcome metrics and motion measurements except for the SPADI score after TSA [5•].

Female and male patients appear to be at risk of different postoperative complications, although the overall complication rate seems to be comparable. Female patients may be more likely to experience implant loosening and periprosthetic fracture while male patients may be more likely to sustain periprosthetic infection and undergo revision surgery. Okoroha et al. hypothesized that this difference is partially explained by increased fall rate in female patients leading to mechanical complications $[17 \cdot \bullet]$.

The current literature on gender-based differences in shoulder arthroplasty is limited in scope and often conflicting in conclusions. Further research is needed to comprehensively evaluate the influence of gender on shoulder arthroplasty, especially as the incidence of shoulder arthroplasty continues to increase. With a deeper understanding of how gender specifically impacts patients undergoing shoulder arthroplasty at the preoperative and postoperative levels, we can better inform patient expectations and decision-making, and ultimately improve patient outcomes.

\section{Compliance with Ethical Standards}

Conflict of Interest Nicole J. Hung declares that she has no conflict of interest. Stephanie E. Wong has received speaker honorarium from DJO, Inc.

Human and Animal Rights and Informed Consent This article does not contain any studies with human or animal subjects performed by any of the authors.

Open Access This article is licensed under a Creative Commons Attribution 4.0 International License, which permits use, sharing, adaptation, distribution and reproduction in any medium or format, as long as you give appropriate credit to the original author(s) and the source, provide a link to the Creative Commons licence, and indicate if changes were made. The images or other third party material in this article are included in the article's Creative Commons licence, unless indicated otherwise in a credit line to the material. If material is not included in the article's Creative Commons licence and your intended use is not permitted by statutory regulation or exceeds the permitted use, you will need to obtain permission directly from the copyright holder. To view a copy of this licence, visit http://creativecommons.org/licenses/by/4.0/. 


\section{References}

Papers of particular interest, published recently, have been highlighted as:

- Of importance

•- Of major importance

1. Péan J. De la prosthèe osseuse. Gaz Hôp Paris. 1897;70:594.

2. Neer CS 2nd. Articular replacement for the humeral head. J Bone Joint Surg Am. 1955;37-A(2):215-28.

3. Kim SH, Wise BL, Zhang Y, Szabo RM. Increasing incidence of shoulder arthroplasty in the United States. J Bone Joint Surg Am. 2011;93(24):2249-54. https://doi.org/10.2106/JBJS.J.01994.

4. Drake GN, O'Connor DP, Edwards TB. Indications for reverse total shoulder arthroplasty in rotator cuff disease. Clin Orthop Relat Res. 2010;468(6):1526-33. https://doi.org/10.1007/s11999-009-11889.

5. Simovitch R, Flurin PH, Wright T, Zuckerman JD, Roche CP. Quantifying success after total shoulder arthroplasty: the substantial clinical benefit. J Shoulder Elbow Surg. 2018;27(5):903-11. https://doi.org/10.1016/j.jse.2017.12.014. Epub 2018 Feb 15. In this retrospective study of patients undergoing TSA and RTSA who were prospectively enrolled in a multicenter database, female sex was associated with lower MCID for the SST, UCLA, ASES, and Constant but not for the SPADI test compared to male sex. MCID was also found to be lower for all planes of motion

6. Simovitch R, Flurin PH, Marczuk Y, Friedman R, Wrigh TW, Zuckerman JD, Roche CP. Rate of improvement in clinical outcomes with anatomic and reverse total shoulder arthroplasty. Bull Hosp Jt Dis. 2013;2015(73 Suppl 1):S111-7.

7. Kerr R, Resnick D, Pineda C, Haghighi P. Osteoarthritis of the glenohumeral joint: a radiologic-pathologic study. AJR Am J Roentgenol. 1985;144(5):967-72. https://doi.org/10.2214/ajr.144. 5.967.

8. Petersson CJ. Degeneration of the gleno-humeral joint. An anatomical study. Acta Orthop Scand. 1983;54(2):277-83. https://doi.org/ 10.3109/17453678308996570.

9. Padegimas EM, Maltenfort M, Lazarus MD, Ramsey ML, Williams GR, Namdari S. Future patient demand for shoulder arthroplasty by younger patients: national projections. Clin Orthop Relat Res. 2015;473(6):1860-7. https://doi.org/10.1007/ s11999-015-4231-z. Epub 2015 Mar 11

10. Day JS, Lau E, Ong KL, Williams GR, Ramsey ML, Kurtz SM. Prevalence and projections of total shoulder and elbow arthroplasty in the United States to 2015. J Shoulder Elbow Surg. 2010;19(8): 1115-20. https://doi.org/10.1016/j.jse.2010.02.009. Epub 2010 Jun 15

11. Hawker GA, Wright JG, Coyte PC, Williams JI, Harvey B, Glazier $\mathrm{R}$, Badley EM. Differences between men and women in the rate of use of hip and knee arthroplasty. N Engl J Med. 2000;342(14): 1016-22. https://doi.org/10.1056/NEJM200004063421405.

12. Lim JB, Chi CH, Lo LE, Lo WT, Chia SL, Yeo SJ, Chin PL, Tay KJ, Lo NN. Gender difference in outcome after total knee replacement. J Orthop Surg (Hong Kong). 2015;23(2):194-7. https://doi. org/10.1177/230949901502300216.

13. Volkmann ER, FitzGerald JD. Reducing gender disparities in posttotal knee arthroplasty expectations through a decision aid. BMC Musculoskelet Disord. 2015;16(1):16. https://doi.org/10.1186/ s12891-015-0473-x.

14. Singh JA, O'Byrne M, Harmsen S, Lewallen D. Predictors of moderate-severe functional limitation after primary total knee arthroplasty (TKA): 4701 TKAs at 2-years and 2935 TKAs at 5- years. Osteoarthritis Cartilage. 2010;18(4):515-21. https://doi.org/ 10.1016/j.joca.2009.12.001. Epub 2009 Dec 21

15. Kennedy D, Stratford PW, Pagura SM, Walsh M, Woodhouse LJ. Comparison of gender and group differences in self-report and physical performance measures in total hip and knee arthroplasty candidates. J Arthroplasty. 2002;17(1):70-7. https://doi.org/10. 1054/arth.2002.29324.

16. Jawa A, Dasti U, Brown A, Grannatt K, Miller S. Gender differences in expectations and outcomes for total shoulder arthroplasty: a prospective cohort study. J Shoulder Elbow Surg. 2016;25(8): 1323-7. https://doi.org/10.1016/j.jse.2016.03.003. Epub 2016 Jun 7

17.• Okoroha KR, Muh S, Gabbard M, Evans T, Roche C, Flurin PH, Wright TW, Zuckerman JD. Early outcomes of shoulder arthroplasty according to sex. JSES Open Access. 2019;3(1):437. https://doi.org/10.1016/j.jses.2018.12.001. In this retrospective review of patients undergoing TSA and RTSA $(n=2364)$, authors assessed sex-based outcomes at minimum 2-year follow-up. Female patients were found to undergo shoulder arthroplasty at an older age with lower preoperative ASES, Constant, and SST scores. Female patients were also found to have a higher incidence of implant loosening and periprosthetic fractures while male patients had a higher incidence of periprosthetic infection. There was no difference in length of stay between female and male patients

18.• Wong SE, Pitcher AA, Ding DY, Cashman N, Zhang AL, Ma CB, Feeley BT. The effect of patient gender on outcomes after reverse total shoulder arthroplasty. J Shoulder Elbow Surg. 2017;26(11): 1889-96. https://doi.org/10.1016/j.jse.2017.07.013. Epub 2017 Sep 11. In this prospective, single center study, authors assessed whether gender influences preoperative disability and outcome measures after RTSA. Female and male patients were found to have similar levels of preoperative disability and similar improvements in pain and ROM after RTSA; however, male patients had higher ASES function and SF-12 scores postoperatively

19.• Friedman RJ, Cheung EV, Flurin PH, Wright T, Simovitch RW, Bolch C, Roche CP, Zuckerman JD. Are age and patient gender associated with different rates and magnitudes of clinical improvement after reverse shoulder arthroplasty? Clin Orthop Relat Res. 2018;476(6):1264-73. https://doi.org/10.1007/s 11999. 0000000000000270 . Authors of this retrospective review of 660 patients who underwent RTSA found that female patients had lower baseline functional scores and reduced ROM compared to male patients before RTSA. Male sex was also found to be associated with higher postoperative functional outcome scores and better ROM in all planes of motion except for external rotation

20. Henn RF 3rd, Ghomrawi H, Rutledge JR, Mazumdar M, Mancuso CA, Marx RG. Preoperative patient expectations of total shoulder arthroplasty. J Bone Joint Surg Am. 2011;93(22):2110-5. https:// doi.org/10.2106/JBJS.J.01114.

21. Mancuso CA, Altchek DW, Craig EV, Jones EC, Robbins L, Warren RF, Williams-Russo P. Patients' expectations of shoulder surgery. J Shoulder Elbow Surg. 2002;11(6):541-9. https://doi.org/ $10.1067 / \mathrm{mse} .2002 .126764$.

22. Singh JA, Sperling JW, Cofield RH. Cardiopulmonary complications after primary shoulder arthroplasty: a cohort study. Semin Arthritis Rheum. 2012;41(5):689-97. https://doi.org/10.1016/j. semarthrit.2011.09.003. Epub 2011 Nov 4

23. Xu S, Baker DK, Woods JC, Brabston EW 3rd, Ponce BA. Risk factors for early readmission after anatomical or reverse total shoulder arthroplasty. Am J Orthop (Belle Mead NJ). 2016;45(6):E38692.

24. Dunn JC, Lanzi J, Kusnezov N, Bader J, Waterman BR, Belmont PJ Jr. Predictors of length of stay after elective total shoulder 
arthroplasty in the United States. J Shoulder Elbow Surg. 2015;24(5):754-9. https://doi.org/10.1016/j.jse.2014.11.042. Epub 2015 Jan 13

25. Menendez ME, Baker DK, Fryberger CT, Ponce BA. Predictors of extended length of stay after elective shoulder arthroplasty. J Shoulder Elbow Surg. 2015;24(10):1527-33. https://doi.org/10. 1016/j.jse.2015.02.014. Epub 2015 Apr 10

26. Matsen FA 3rd, Li N, Gao H, Yuan S, Russ SM, Sampson PD. Factors affecting length of stay, readmission, and revision after shoulder arthroplasty: a population-based study. J Bone Joint Surg Am. 2015;97(15):1255-63. https://doi.org/10.2106/JBJS.N. 01107. Erratum in: J Bone Joint Surg Am. 2015 Sep 2;97(17):e60

27. Gruson KI, Accousti KJ, Parsons BO, Pillai G, Flatow EL. Transfusion after shoulder arthroplasty: an analysis of rates and risk factors. J Shoulder Elbow Surg. 2009;18(2):225-30. https://doi. org/10.1016/j.jse.2008.08.005. Epub 2008 Dec 31

28. Donigan JA, Frisella WA, Haase D, Dolan L, Wolf B, Belmont PJ Jr. Pre-operative and intra-operative factors related to shoulder arthroplasty outcomes. Iowa Orthop J. 2009;29:60-6.

29. Matsen FA 3rd, Antoniou J, Rozencwaig R, Campbell B, Smith KL. Correlates with comfort and function after total shoulder arthroplasty for degenerative joint disease. J Shoulder Elbow Surg. 2000;9(6):465-9. https://doi.org/10.1067/mse.2000.109323.

30. Hollatz MF, Stang A. Nationwide shoulder arthroplasty rates and revision burden in Germany: analysis of the national hospitalization data 2005 to 2006. J Shoulder Elbow Surg. 2014;23(11):e267-74. https://doi.org/10.1016/j.jse.2013.12.008. Epub 2014 Mar 4

31. Singh JA, Sperling JW, Cofield RH. Revision surgery following total shoulder arthroplasty: analysis of 2588 shoulders over three decades (1976 to 2008). J Bone Joint Surg Br. 2011;93(11):1513-7. https://doi.org/10.1302/0301-620X.93B11.26938.

32. Karlson EW, Daltroy LH, Liang MH, Eaton HE, Katz JN. Gender differences in patient preferences may underlie differential utilization of elective surgery. Am J Med. 1997;102(6):524-30. https:// doi.org/10.1016/s0002-9343(97)00050-8.

33. Chang HJ, Mehta PS, Rosenberg A, Scrimshaw SC. Concerns of patients actively contemplating total knee replacement: differences by race and gender. Arthritis Rheum. 2004;51(1):117-23. https:// doi.org/10.1002/art.20073.

34. Othman H, Khambatta S, Seth M, Lalonde TA, Rosman HS, Gurm HS, Mehta RH. Differences in sex-related bleeding and outcomes after percutaneous coronary intervention: insights from the Blue Cross Blue Shield of Michigan Cardiovascular Consortium (BMC2) registry. Am Heart J. 2014;168(4):552-9. https://doi.org/ 10.1016/j.ahj.2014.07.012. Epub 2014 Jul 25

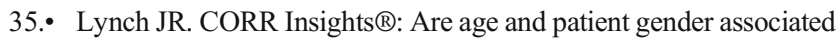
with different rates and magnitudes of clinical improvement after reverse shoulder arthroplasty? Clin Orthop Relat Res. 2018;476(6): 1274-5. https://doi.org/10.1097/01.blo.0000533613.25243.1c. In this response to Friedman et al.'s paper (19), Lynch suggests that next research steps regarding gender and shoulder arthroplasty should include a formal, gender-based analysis of reliability, responsiveness, variability, and validity of commonly used patient reported outcome measures

Publisher's note Springer Nature remains neutral with regard to jurisdictional claims in published maps and institutional affiliations. 